\title{
Hierarchical cluster analysis of myoepithelial/ basal cell markers in adenoid cystic carcinoma and polymorphous low-grade adenocarcinoma
}

\author{
Manju L Prasad ${ }^{1,5}$, Catalin C Barbacioru², Yeshwant B Rawal ${ }^{3}$, Omar Husein ${ }^{4}$ and \\ Ping Wen ${ }^{1}$ \\ ${ }^{1}$ Department of Pathology, Ohio State University, Columbus, OH, USA; ${ }^{2}$ Applied Biosystems, Foster City, CA, \\ USA; ${ }^{3}$ College of Dentistry, Ohio State University, Columbus, OH, USA; ${ }^{4}$ Head \& Neck Surgery, Ohio State \\ University, Columbus, OH, USA and ${ }^{5}$ UMass Memorial Medical Center, Worcester, MA, USA
}

\begin{abstract}
Distinguishing adenoid cystic carcinoma from polymorphous low-grade adenocarcinoma of the salivary glands is important for their management. We studied the expression of several myoepithelial and basal/stem cell markers (smooth muscle actin, calponin, smooth muscle myosin heavy chain, metallothionein, maspin, and p63) by immunohistochemistry in 23 adenoid cystic carcinoma and 24 polymorphous low-grade adenocarcinoma, to identify the most useful marker or combination of markers that may help their diagnoses. The results were analyzed using hierarchical cluster analysis and $\chi^{2}$ test for trend. We noted diffuse expression of smooth muscle actin in 20 adenoid cystic carcinoma vs one polymorphous low-grade adenocarcinoma $(P<0.0001)$, calponin in 15 adenoid cystic carcinoma vs one polymorphous low-grade adenocarcinoma $(P<0.0001)$, smooth muscle myosin heavy chain in $\mathbf{1 5}$ adenoid cystic carcinoma vs one polymorphous low-grade adenocarcinoma $(P=0.001)$, metallothionein in 22 adenoid cystic carcinoma vs eight polymorphous low-grade adenocarcinoma $(P<0.001)$, maspin in 22 adenoid cystic carcinoma vs 14 polymorphous low-grade adenocarcinoma, and p63 in 21 adenoid cystic carcinoma vs 14 polymorphous low-grade adenocarcinoma. Hierarchical clustering of smooth muscle actin, calponin, smooth muscle myosin heavy chain, and metallothionein was virtually identical $(\kappa \leq 0.0035)$, suggesting no significant advantage to their use in combination than individually. Diffuse smooth muscle actin expression showed the highest accuracy $(91.5 \%)$ and positive predictive value $(95.2 \%)$ for adenoid cystic carcinoma. Thus, diffuse expression of smooth muscle actin, calponin, smooth muscle myosin heavy chain, and metallothionein was highly predictive of adenoid cystic carcinoma, whereas maspin and p63 were frequently expressed in both tumors. In differentiating adenoid cystic carcinoma from polymorphous low-grade adenocarcinoma, smooth muscle actin as a single ancillary test in support of the histological findings, appears to be as efficient as multiple immunohistochemical tests.
\end{abstract}

Modern Pathology (2008) 21, 105-114; doi:10.1038/modpathol.3800983; published online 14 December 2007

Keywords: adenoid cystic carcinoma; polymorphous low grade adenocarcinoma; immunohistochemistry; maspin; p63; metallothionein; calponin

Adenoid cystic carcinoma accounts for $25 \%$ of all malignant salivary gland tumors. It is a relentlessly growing malignancy that frequently recurs and metastasizes. Surgical removal is challenging and positive margins are frequent, requiring adjuvant radiotherapy. Adenoid cystic carcinoma has significant histologic similarities with polymorphous low-grade adenocarcinoma that includes tubular

Correspondence: Dr ML Prasad, MD, Department of Pathology, UMass Memorial Medical Center, 3-Biotech, 1-Innovation Dr, Worcester, MA 1605, USA.

E-mail: prasadm@ummhc.org

Received 24 January 2007; revised 29 October 2007; accepted 31

October 2007; published online 14 December 2007 and cribriform growth patterns, infiltrating borders, and perineural invasion. However, in contrast to adenoid cystic carcinoma, polymorphous low-grade adenocarcinoma has an excellent cure rate after complete excision, infrequent recurrences, and rare metastasis. In a series of 164 polymorphous lowgrade adenocarcinoma, $97.6 \%$ of patients were either alive or dead without evidence of recurrent disease after treatment with surgical excision only at 115 months after presentation. ${ }^{1}$ Therefore, the precise diagnosis of adenoid cystic carcinoma and polymorphous low-grade adenocarcinoma is essential for their appropriate management. Ancillary methods, for example, immunohistochemistry with smooth muscle/myoepithelial markers, basal/stem 
cell markers, proliferation markers, and c-kit have been employed to help distinguish them with variable results. ${ }^{2-4}$ Recently, several new myoepithelial and basal cell markers have become available, but their utility in distinguishing adenoid cystic carcinoma from polymorphous low-grade adenocarcinoma is unknown. We studied the immunohistochemical-staining pattern of two relatively new markers, metallothionein and maspin in adenoid cystic carcinoma and polymorphous low-grade adenocarcinoma, and compared the results with some well-known myoepithelial cell markers.

Metallothioneins are a family of low-molecular weight, metal-binding, cysteine-rich intracellular proteins coded by the MT genes. The induction and expression of these proteins have been associated with oxidative stress and apoptosis, and appear to be cell-type specific. Metallothionein is a cytosolic protein in resting cells, but it can be translocated transiently to the nucleus during cell proliferation and differentiation. ${ }^{5}$ Strong expression of metallothionein is seen in simple epithelia, basal, and myoepithelial cells, specially in normal and neoplastic breast tissue. ${ }^{6}$ Increased expression of the MT genes is also noted in tumors of the colon, kidney, liver, lung, nasopharynx, ovary, prostate, salivary gland, testis, thyroid, and urinary bladder. $^{7,8}$

Maspin is a mammary serine protease inhibitor with homology to members of the serpin family of protease inhibitors. It is a tumor suppressor gene protein that inhibits tumor cell invasion, motility, proliferation, and angiogenesis. ${ }^{9}$ It is expressed in normal human mammary epithelial and myoepithelial cells. The mammary myoepithelial cells and prostatic basal cells stain intensely for maspin by immunohistochemistry. Maspin expression is also noted in squamous and glandular epithelia, endometrium, and testicular germ cells. p63, a homolog of p53, is expressed in embryonic ectoderm, basal/ stem cells of stratified epithelium, and myoepithelial cells. p63 expression is consistently lost in invasive adenocarcinoma of prostate and breast but preserved in squamous, transitional, and basal cell carcinoma.

In addition, we studied the traditional markers of myoepithelial cells, for example, $\alpha$-smooth muscle actin, calponin, and smooth muscle myosin heavy chain, that react with contractile cytoskeletal proteins associated with myoid differentiation. ${ }^{10}$ The expression of these proteins in salivary gland tumors of myoepithelial differentiation and their absence in non-myoepithelial tumors, for example, mucoepidermoid carcinoma, acinic cell carcinoma, Warthin's tumor, squamous cell carcinoma, polymorphous low-grade adenocarcinoma is well documented. ${ }^{11}$ Here, we evaluate some traditional cytoplasmic (smooth muscle actin, calponin, smooth muscle myosin heavy chain) and some relatively less known, nuclear or predominantly nuclear basal cell markers (metallothionein, maspin, p63) to determine the most efficient marker or combination of markers in discriminating adenoid cystic carcinoma from polymorphous low-grade adenocarcinoma.

\section{Materials and methods}

\section{Tumor Samples}

Tumors with a diagnosis of adenoid cystic carcinoma and polymorphous low-grade adenocarcinoma were identified from the archives of the Departments of Pathology, and Oral and Maxillofacial Pathology, College of Dentistry, the Ohio State University Medical Center. Cases were reviewed by at least two pathologists trained in head and neck (MLP) and oral and maxillofacial pathology (YBR). Clinical and follow-up information was reviewed when available.

\section{Immunohistochemistry}

Immunohistochemistry was performed on $4 \mu$ thick sections of formalin-fixed, paraffin-embedded tissues using the labeled streptavidin-biotin complex system (LSAB2, Dako) in a Dako Autostainer (DakoCytomation, Carpinteria, CA, USA). Heatinduced antigen retrieval was carried out by steaming unstained sections in Target Retrieval Solution (Dako TRS pH 6.1) for $30 \mathrm{~min}$. The incubation with monoclonal primary antibodies varied from 30 to $60 \mathrm{~min}$ at room temperature (Table 1). Appropriate positive (normal breast) and negative controls were used. In most tumor samples, non-neoplastic salivary glands adjacent to the tumor provided a built-in positive control with decoration of myoepithelial

Table 1 Primary antibodies

\begin{tabular}{llc}
\hline Primary antibodies against & Ag retrieval & Dilution/concentration \\
\hline SMA & Dako TRS (pH 6.1) & $1: 75$ \\
Calponin & Dako TRS (pH 6.1) & $1: 100$ \\
SMMS & Dako TRS (pH 6.1) & $1: 75$ \\
Metallothionein (MT) & Dako TRS (pH 6.1) & $1: 800$ \\
Maspin & Dako TRS (pH 6.1) & $1: 800$ \\
p63 & Dako TRS (pH 6.1) & Dako, SMLP \\
& & Dako, E9 \\
\hline
\end{tabular}

Incubation time: $60 \mathrm{~min}$ for maspin and $30 \mathrm{~min}$ for all others, at room temperature. 
cells by all markers. The immunostaining was cytoplasmic for smooth muscle actin, calponin, and smooth muscle myosin heavy chain, nuclear for p63, and predominantly nuclear with some cytoplasmic staining for metallothionein and maspin.

The immunostains were interpreted as positive when at least $10 \%$ of cells expressed the antigen. The positive expression was further categorized into focal $(10-<33 \%)$ and diffuse $(\geq 33 \%)$. No expression or expression in less than $10 \%$ of tumor cells was considered negative.

\section{Statistical Analysis}

The statistical analysis focused on two aspects: (i) the association of the protein expression with adenoid cystic carcinoma and polymorphous lowgrade adenocarcinoma, and (ii) the concordance between the six protein markers. We used $\chi^{2}$ test for trend to see if there is an increasing (or decreasing) trend in the proportion of adenoid cystic carcinoma samples within the diffuse, focal, and no expression categories for each protein (significant if $P<0.01$ ). The sensitivity (true positive/(true positive + false negative)), specificity (true negative/(true negative + false positive)), positive predictive value (true positive/(true + false positive)), and accuracy ((true positive + true negative)/All cases (true positive + false positive + true negative + false negative)) were calculated for each protein for the diagnosis of adenoid cystic carcinoma.

In the second part of analysis, the concordance between proteins was analyzed using $\kappa$ statistics, which represents the proportion of agreements after excluding chance agreement. Pairwise $\kappa$ values were calculated using Bonferroni correction for multiple testing. Using $1-\kappa$ statistics as a measure of dissimilarity, $\kappa$ values equal to or below 0.0035 suggested a significant association between pairs of proteins. Hierarchical clustering of proteins was based on Ward's minimum variance method, which aims in finding compact, spherical clusters using $\kappa$ statistics distance metric. All analysis was carried out using the statistical software package $\mathrm{R}$ (http://www. r-project.org). ${ }^{12}$

\section{Results}

Table 2 shows the age and sex distribution, and site of 23 adenoid cystic carcinoma and 24 polymorphous low-grade adenocarcinoma included in the study. Nine adenoid cystic carcinomas involved the major salivary glands, 13 involved minor salivary or seromucous glands, and one involved the Bartholin's gland. Clinical and follow-up information was available in 21 adenoid cystic carcinomas. Using the staging criteria recommended by the American Joint Committee on Cancer, 10 adenoid cystic carcinomas were $T_{2}$, two were $T_{3}$, and nine were $T_{4}$.
Table 2 Clinical features

\begin{tabular}{|c|c|c|}
\hline Tumor type & $\begin{array}{l}\text { Median age (range) } \\
\text { (in years) }\end{array}$ & $\begin{array}{c}\text { Sex } \\
\text { Females/males }\end{array}$ \\
\hline $\begin{array}{l}\text { ACC }(\mathrm{N}=23 \text { ) } \\
\text { (Parotid gland } 6 \text {, } \\
\text { submandibular gland } 3 \text {, } \\
\text { oral cavity } 2 \text {, nasal/ } \\
\text { paranasal sinuses } 6 \text {, ear } \\
2 \text {, trachea 2, larynx } 1 \text {, } \\
\text { Bartholin's gland } 1 \text { ) }\end{array}$ & $52(23-82)$ & $13 \mathrm{~F}: 10 \mathrm{M}$ \\
\hline $\begin{array}{l}P L G A(\mathrm{~N}=24) \\
\text { (Palate 19, lip 5, buccal } \\
\text { mucosa 2) }\end{array}$ & $57(32-94)$ & $18 \mathrm{~F}: 6 \mathrm{M}$ \\
\hline
\end{tabular}

ACC, adenoid cystic carcinoma; PLGA, polymorphous low grade adenocarcinoma.

Five adenoid cystic carcinoma patients had nodal metastases $\left(N_{1}=3, N_{2}=2\right)$ and two patients had lung metastases at presentation. Twenty adenoid cystic carcinoma patients were treated with definitive surgery, and 14 received adjuvant radiotherapy. One patient was treated with palliative surgery and radiotherapy. Follow-up varied from less than 1 year to 25 years (median 3.5). Locoregional recurrence developed in 11 adenoid cystic carcinoma patients. Nine adenoid cystic carcinoma patients had distant metastasis $\left(\mathrm{M}_{1}\right)$, two at presentation and seven on follow-up, most frequently to the lungs. Ten adenoid cystic carcinoma patients were alive and free of disease, six were alive with disease and five died of disease. All polymorphous low-grade adenocarcinomas arose in the minor salivary glands of oral cavity. Clinical details and follow-up data were not available in polymorphous low-grade adenocarcinoma cases.

\section{Microscopy}

The adenoid cystic carcinoma showed a predominantly tubulo-cribriform pattern in 20 tumors (Figure 1a and b). The dimorphic tumor cell population was well appreciated in the majority of adenoid cystic carcinoma. In tumor nests and tubules, tumor cells with angulated dark nuclei (myoepithelial type) were disposed adjacent to the basement membrane in peripheral, abluminal distribution, and a second, epithelial type of tumor cells with vesicular nuclei and small distinct or indistinct nucleoli were seen adjacent to lumen. Three adenoid cystic carcinoma showed solid areas comprising $30-80 \%$ of the tumor, admixed with the usual tubulo-cribriform pattern. All three adenoid cystic carcinoma with solid areas and one adenoid cystic carcinoma with predominantly cribriform pattern showed necrosis, that resembled comedotype necrosis in two of them (Figure 1e).

The polymorphous low-grade adenocarcinoma showed a mixed tubulo-cribriform architecture with 

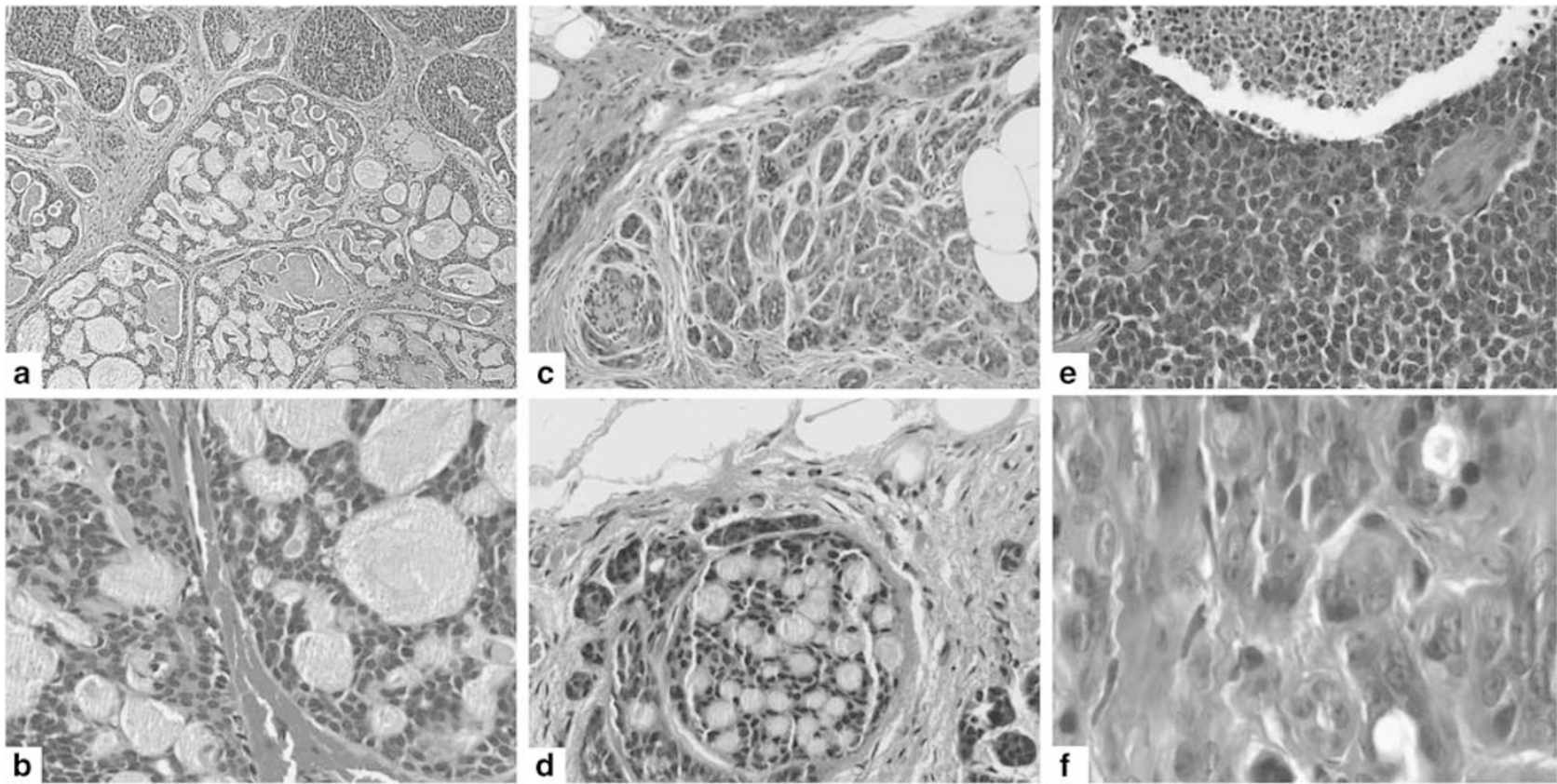

Figure 1 (a and b) Adenoid cystic carcinoma showing cribriform growth pattern and tumor cell nests. (c and d) Polymorphous low-grade adenocarcinoma showing tumor infiltration into fat and perineural space, (c) and cribriform architecture (d). (e) Solid-type adenoid cystic carcinoma with mitoses and central necrosis. (f) Polymorphous low grade adenocarcinoma showing a tubule lined by a single layer of cuboid, monomorphic tumor cells with round, vesicular nuclei containing indistinct nucleoli (same case as in d).

the exception of one tumor that showed a predominantly fascicular pattern with spindling of tumor cells. In contrast to adenoid cystic carcinoma, tubules, and glands were lined by a single layer of monomorphic, small to medium tumor cells with bland, low grade, vesicular nuclei, and occasional indistinct nucleoli (Figure 1f). Tumors were well defined, but had microscopically infiltrative borders, and showed neural invasion (Figure 1c and d). Single filing of tumor cells, trabecular, and targetoid patterns were frequently seen, especially at the periphery of the tumors. Solid, cystic and papillary features, mucin production, and oncocytic change were focally noted. Dystrophic calcification and occasionally, tyrosine crystals were also noted. No necrosis was seen in the polymorphous low-grade adenocarcinoma.

\section{Immunohistochemistry}

The results of immunohistochemistry are summarized in Table 3.

Adenoid cystic carcinoma showed strong and diffuse cytoplasmic expression of smooth muscle actin, calponin, and smooth muscle myosin heavy chain in the peripheral, abluminal tumor cells (Figure 2). The luminal cells showed variable staining from being completely negative to positive. Luminal cells that lined spaces containing basement membrane type or mucinous material were usually positive while those lining true lumina and displaying epithelial features were negative. Variable expression was also seen in cells that were in between the peripheral and the luminal cells (intermediate cells). Similarly, nuclear and cytoplasmic expression of metallothionein and maspin, and nuclear expression of p63 was seen in the peripheral abluminal cells, intermediate cells, and in some luminal cells (Figure 2). The expression of all markers was markedly diminished in adenoid cystic carcinoma with solid areas. Two predominantly solid adenoid cystic carcinoma with necrosis and brisk mitoses showed a single layer of positive cells at the periphery of solid tumor cell nests (Figure 3), with absent or occasional expression in the remaining tumor cells.

In most polymorphous low-grade adenocarcinoma, tumor cells were completely negative or focally positive for smooth muscle actin, calponin, and smooth muscle myosin heavy chain (Figure $2 \mathrm{~d}-\mathrm{f}$ ). Diffuse expression of metallothionein, maspin, and p63 was seen in eight (33\%), $14(58 \%)$ and 14 polymorphous low-grade adenocarcinoma (58\%) respectively, while the expression was negative or focal in the remaining (Table 3). When diffusely expressed, tumor cell nuclei uniformly stained with metallothionein, maspin, and p63, and did not show the zonal distribution pattern seen in adenoid cystic carcinoma (Figure 2j-l).

\section{Statistical Analysis}

Using $\chi^{2}$ test for trend (significant if $P<0.01$ ), we observed a significant association between the 
Table 3 Expression of proteins

\begin{tabular}{|c|c|c|c|c|c|c|c|}
\hline Markers & $A C C \mathrm{n} 23$ & $P L G A$ n 24 & $\mathrm{P}$-value ${ }^{\mathrm{a}}$ & Accuracy & $P P V^{\mathrm{b}}$ & Sensitivity & Specificity $^{\mathrm{b}}$ \\
\hline \multicolumn{8}{|l|}{$S M A$} \\
\hline Total positive & $21(91.3 \%)$ & $4(16.6 \%)$ & & & & & \\
\hline Diffusely positive & $20(87 \%)$ & $1(4.1 \%)$ & $<0.0001$ & $91.5 \%$ & $95.2 \%$ & $87 \%$ & $95.8 \%$ \\
\hline Negative & 2 & 20 & & & & & \\
\hline \multicolumn{8}{|l|}{ Calponin } \\
\hline Total positive & $21(91.3 \%)$ & $5(20.8 \%)$ & & & & & \\
\hline Diffusely positive & $15(65.2 \%)$ & $1(4.1 \%)$ & $<0.0001$ & $80.9 \%$ & $93.8 \%$ & $65.2 \%$ & $95.8 \%$ \\
\hline Negative & 2 & 19 & & & & & \\
\hline \multicolumn{8}{|l|}{ SMMS } \\
\hline Total positive & $18(78 \%)$ & $11(45.8 \%)$ & & & & & \\
\hline Diffusely positive & $15(65.2 \%)$ & $1(4.1 \%)$ & $=0.001$ & $80.9 \%$ & $93.8 \%$ & $65.2 \%$ & $95.8 \%$ \\
\hline Negative & 5 & 13 & & & & & \\
\hline \multicolumn{8}{|l|}{ Metallothionein } \\
\hline Total positive & $23(100 \%)$ & $20(83 \%)$ & & & & & \\
\hline Diffusely positive & $22(95.7 \%)$ & $8(33.3 \%)$ & $<0.001$ & $80.9 \%$ & $73.3 \%$ & $95.7 \%$ & $66.7 \%$ \\
\hline Negative & 0 & 4 & & & & & \\
\hline \multicolumn{8}{|l|}{ Maspin } \\
\hline Total positive & $23(100 \%)$ & $22(91.6 \%)$ & & & & & \\
\hline Diffusely positive & $22(95.7 \%)$ & $14(58.3 \%)$ & 0.014 & $68.1 \%$ & $61 \%$ & $95.7 \%$ & $41.7 \%$ \\
\hline Negative & 0 & 2 & & & & & \\
\hline \multicolumn{8}{|l|}{ p63 } \\
\hline Total positive & $22(95.6 \%)$ & $20(83 \%)$ & & & & & \\
\hline Diffusely positive & $21(91.3 \%)$ & $14(58.3 \%)$ & 0.054 & $66 \%$ & $60 \%$ & $91.3 \%$ & $41.7 \%$ \\
\hline Negative & 1 & 4 & & & & & \\
\hline
\end{tabular}

ACC, adenoid cystic carcinoma; PLGA, polymorphous low-grade adenocarcinoma; PPV, positive predictive value.

${ }^{a} \chi^{2}$ for trend (significant if $P<0.01$ ) calculated for diffuse expression (in bold).

${ }^{\mathrm{b}}$ Using only diffuse expression as positive for the diagnosis of ACC.

expression of smooth muscle actin, calponin, smooth muscle myosin heavy chain, and metallothionein in more tumor cells in adenoid cystic carcinoma than polymorphous low-grade adenocarcinoma ( $P$-values $\leq 0.001$, Table 3$)$. Although maspin and p63 expressions were also more diffuse in adenoid cystic carcinoma than in polymorphous low-grade adenocarcinoma, this difference was not significant.

The expression profile of tumor samples showed the more frequent, diffuse expression of smooth muscle actin, calponin, smooth muscle myosin heavy chain, and metallothionein in adenoid cystic carcinoma compared to polymorphous low-grade adenocarcinoma (Figure 4). These four proteins together discriminated well between adenoid cystic carcinoma and polymorphous low-grade adenocarcinoma as seen in the dendrogram in Figure 5. One adenoid cystic carcinoma (ACC16), a $2.4 \mathrm{~cm}$ mass in the soft palate of a 74-year-old woman, clustered with polymorphous low-grade adenocarcinoma, and was subsequently reclassified as polymorphous low-grade adenocarcinoma in consultation with oral and maxillofacial pathologists who were blinded to the results of this study. Three polymorphous low-grade adenocarcinomas (PLGA4, PLGA5, and PLGA9) clustered with adenoid cystic carcinoma, and were reviewed by an expert head and neck pathologist blinded to the results of the cluster analysis. The expert reclassified PLGA4 as adenoid cystic carcinoma but PLGA5 and PLGA9 were reaffirmed to be polymorphous low-grade adenocarcinoma. PLGA5 had a fascicular architecture with a prominent spindle-cell component that was positive with myoid myoepithelial markers. PLGA9 had the characteristic tubulocystic architecture of polymorphous low-grade adenocarcinoma with trabecular and infiltrative growth pattern, and showed perineural invasion.

Table 4 shows the concordance between protein pairs among all six proteins using $\kappa$ statistics. Significant concordance $(\kappa \leq 0.0035)$ was seen between pairs of proteins in one group (smooth muscle actin, calponin, smooth muscle myosin heavy chain, and metallothionein), and between maspin and p63 in another group $(\kappa=0.0028)$. Hierarchical clustering of the protein markers showed virtually identical expression of smooth muscle actin, calponin, smooth muscle myosin heavy chain, and metallothionein in one cluster, and that of maspin and p63 in another cluster (Figure 6). This indicates that using one marker in a cluster provides the same information as using multiple markers from the same cluster due to the similarities in their expression. If only diffuse expression is considered positive (and expression in $<33 \%$ of tumor cells 


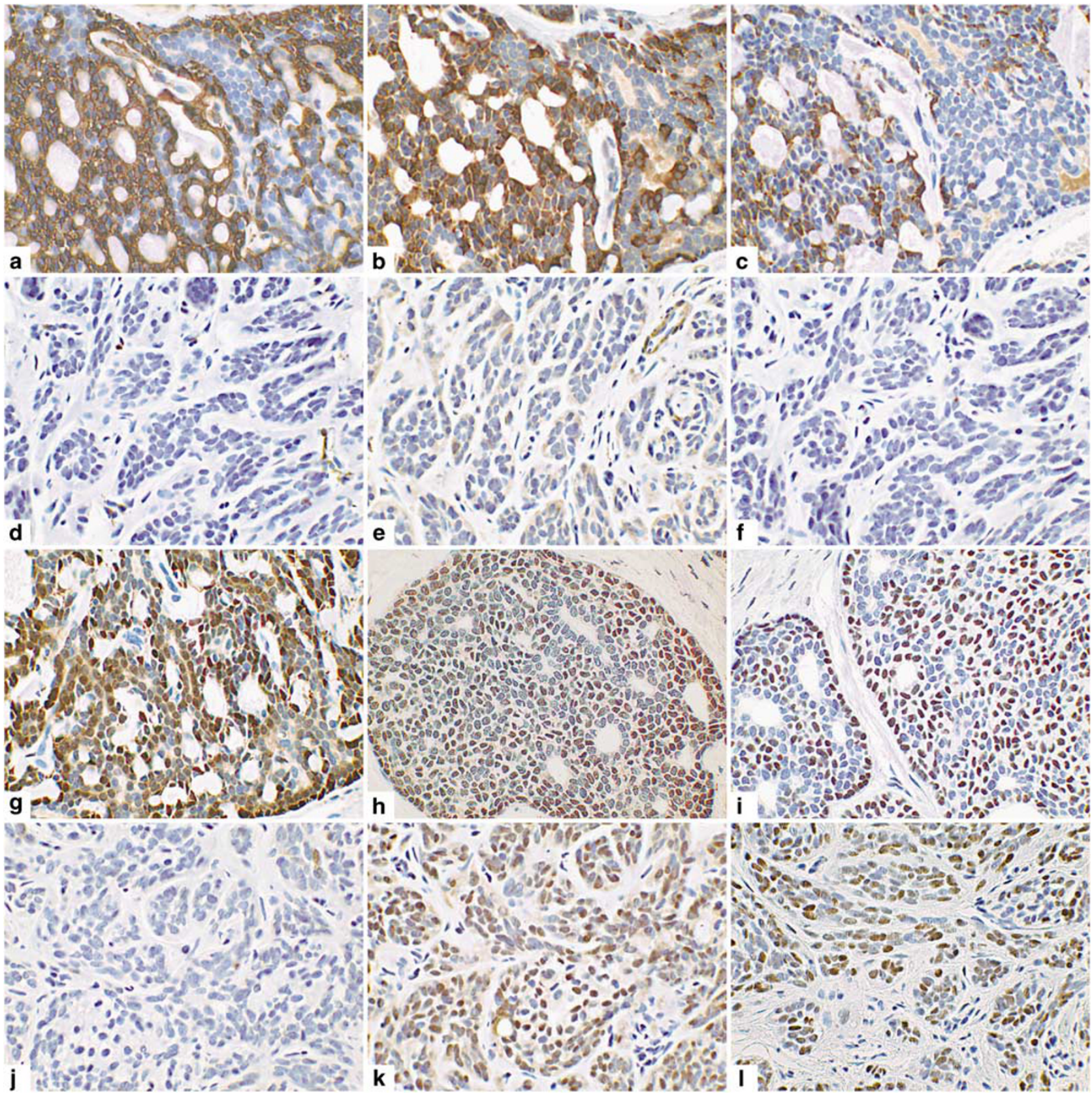

Figure 2 Immunohistochemistry showing expression of smooth muscle actin (a adenoid cystic carcinoma (ACC)-diffusely positive, d polymorphous low-grade adenocarcinoma (PLGA)-negative) calponin (b ACC-diffusely positive, e PLGA-negative), smooth muscle myosin heavy chain (c ACC-positive, f PLGA-negative), metallothionein (g ACC-diffusely positive, $\mathbf{j}$ PLGA-negative), maspin (h ACCpositive, k PLGA-positive), and p63 (i ACC-positive, l PLGA-positive). Smooth muscle actin, calponin, and smooth muscle myosin heavy chain show cytoplasmic staining, metallothionein and maspin show nuclear and cytoplasmic staining, and p63 shows nuclear staining reaction only.

as negative), then smooth muscle actin shows the highest accuracy $(91.5 \%)$, and positive predictive value (95\%) for adenoid cystic carcinoma among all proteins (Table 3 ).

\section{Discussion}

The new World Health Organization's Blue Book series on 'Classification of Tumors: Pathology and
Genetics of Head and Neck Tumors' defines adenoid cystic carcinoma as '... consisting of epithelial and myoepithelial cells...' and polymorphous low-grade adenocarcinoma as 'a malignant epithelial tumor ...' ${ }^{13}$ One of the main differentiating features between the two is identification of a dimorphic tumor cell population with myoepithelial and epithelial-type cells in adenoid cystic carcinoma, and a monomorphic epithelial-type tumor cell population in polymorphous low-grade 

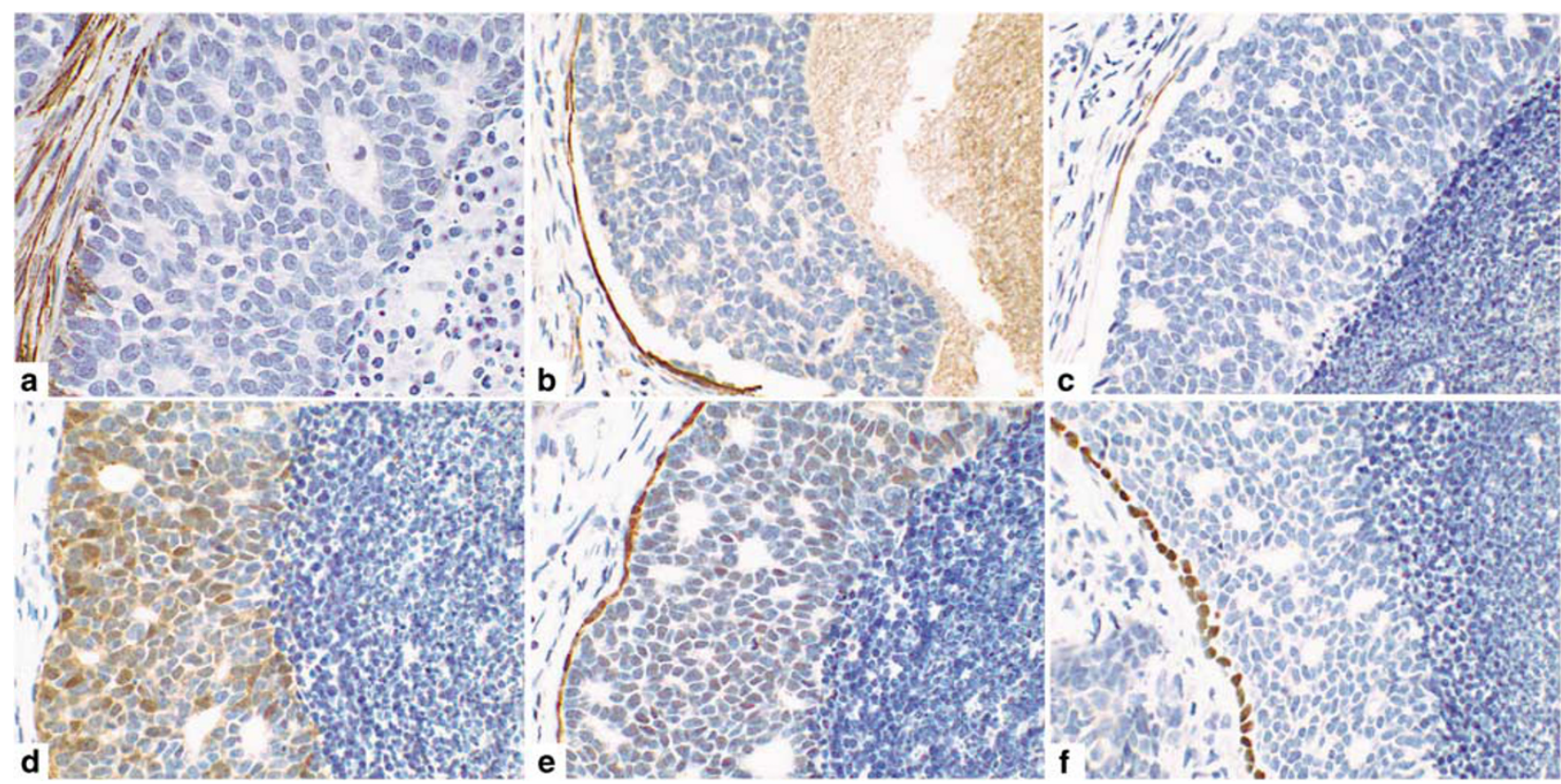

Figure 3 Immunohistochemistry in a solid adenoid cystic carcinoma with necrosis and high mitotic rate (same as Figure 1e). (a) Smooth muscle actin, (b) Calponin, (c) Smooth muscle myosin heavy chain, (d) metallothionein, (e) maspin, (f) p63. Scant expression of myoepithelial/basal cell markers seen at the periphery of tumor cell nests. Tumor necrosis is seen to the right side of all images.

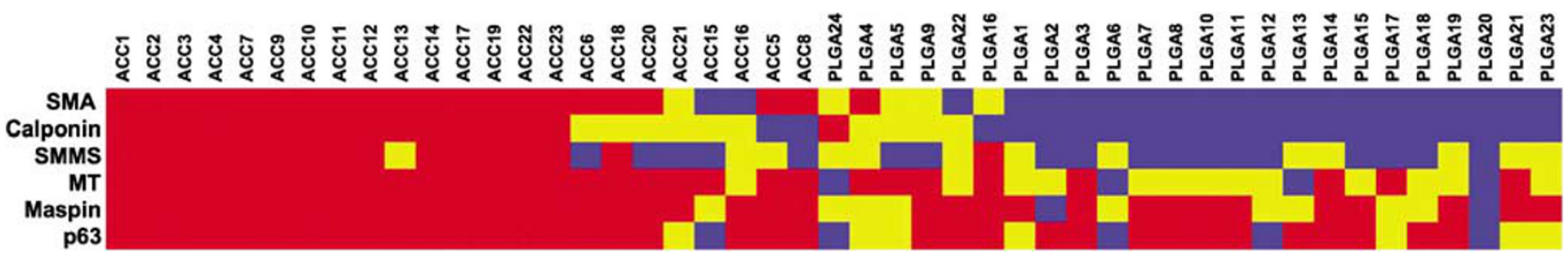

Figure 4 Protein expression profile in all 47 tumor samples. Red: Diffuse, Yellow: Focal, Blue: No expression. Expression of all markers is more frequently diffused in adenoid cystic carcinoma (ACC-left half) as compared to polymorphous low-grade adenocarcinoma (PLGA-right half).

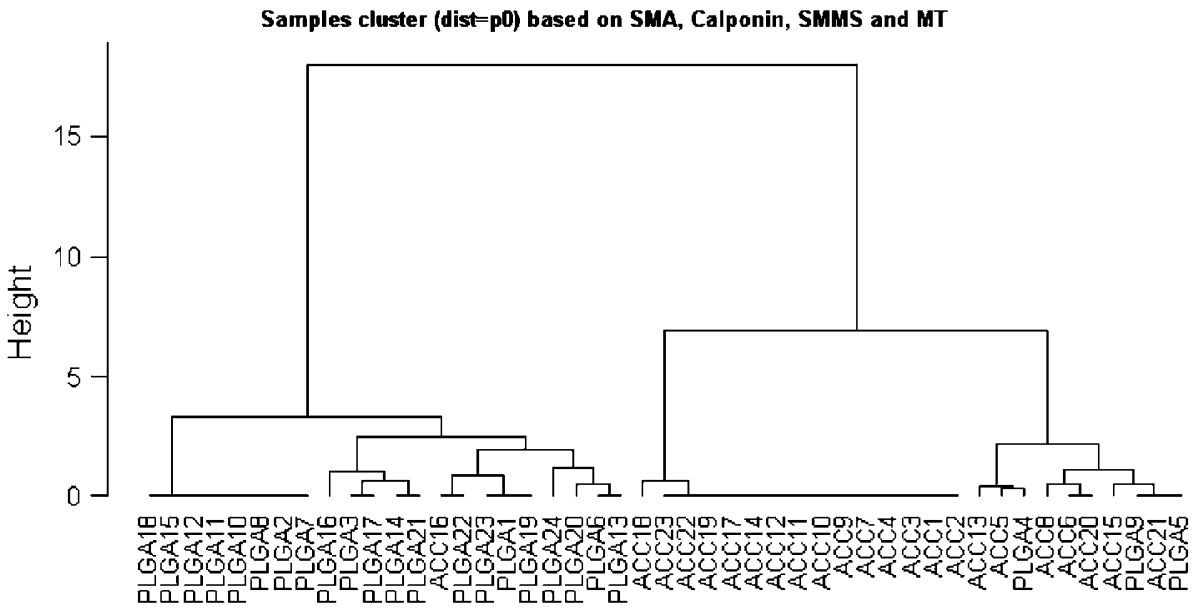

Figure 5 Dendrogram based on the expression of smooth muscle actin, calponin, smooth muscle myosin heavy chain and metallothionein in tumor samples. These four proteins separate polymorphous low-grade adenocarcinoma (PLGA-left side) and adenoid cystic carcinoma (ACC-right side) into two clusters.

adenocarcinoma. The myoepithelial-type tumor cells in adenoid cystic carcinoma are usually peripheral and adjacent to the basement membrane in tumor nests and tubules, and have clear cytoplasm with angulated, hyperchromatic and dense nuclei. The second type of tumor cells in adenoid 
Table 4 Association between protein pairs using $\kappa$ statistics*

\begin{tabular}{lcccccc}
\hline Proteins & $S M A$ & Calponin & $S M M S$ & $M T$ & Maspin & P63 \\
\hline SMA & - & $\mathbf{0 . 0 0 0 0}$ & $\mathbf{0 . 0 0 0 1}$ & $\mathbf{0 . 0 0 0 0}$ & 0.1397 & 0.1657 \\
Calponin & $\mathbf{0 . 0 0 0 0}$ & - & $\mathbf{0 . 0 0 0 0}$ & $\mathbf{0 . 0 0 1 3}$ & 0.3803 & 0.2197 \\
SMMS & $\mathbf{0 . 0 0 0 1}$ & $\mathbf{0 . 0 0 0 0}$ & - & $\mathbf{0 . 0 0 3 5}$ & 0.0866 & 0.0571 \\
MT & $\mathbf{0 . 0 0 0 0}$ & $\mathbf{0 . 0 0 1 3}$ & $\mathbf{0 . 0 0 3 5}$ & - & 0.0184 & 0.0295 \\
Maspin & 0.1397 & 0.3803 & 0.0866 & 0.0184 & - & $\mathbf{0 . 0 0 2 8}$ \\
p63 & 0.1657 & 0.2197 & 0.0571 & 0.0295 & $\mathbf{0 . 0 0 2 8}$ & -
\end{tabular}

${ }^{*} \kappa$ test was used to detect associations between different protein pairs using Bonferroni correction for multiple testing. $\kappa$ values $\leq 0.0035$ suggest a significant association between pairs of proteins (in bold).

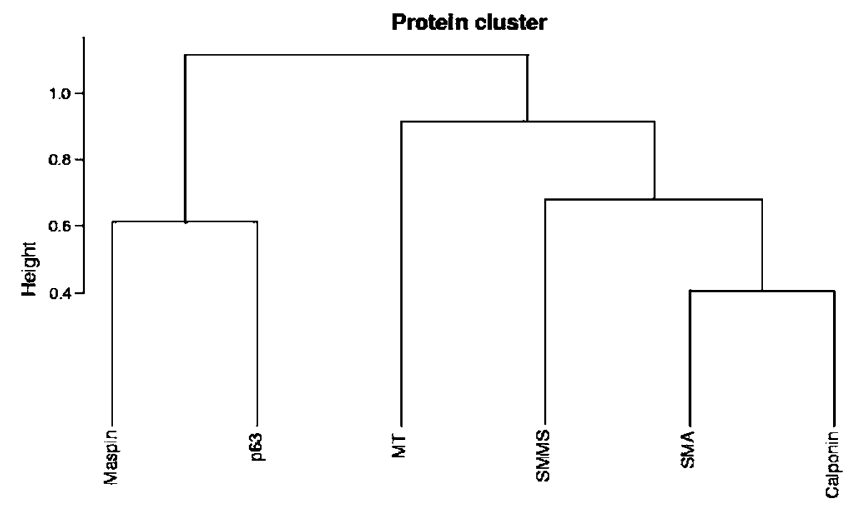

Figure 6 Hierarchical clustering of proteins based on their expression in all 47 tumor samples using $\kappa$ statistic distance metric to assess similarity among the proteins. Smooth muscle actin, calponin, smooth muscle myosin heavy chain, and metallothionein cluster together, while maspin and p63 are in a second cluster.

cystic carcinoma show ductal/epithelial differentiation, are less numerous and arranged towards the lumen away from the basement membrane, and have round nuclei with vesicular chromatin that may contain small nucleoli. Myoepithelial cells share immunophenotypic features with smooth muscle and basal/stem cells. We selected three cytoplasmic myoid smooth muscle proteins associated with actin filaments (smooth muscle actin, calponin, and smooth muscle myosin heavy chain), and three predominantly nuclear proteins associated with basal/stem cells (metallothionein, maspin, and p63). We noted that in adenoid cystic carcinoma the myoid markers (smooth muscle actin, calponin, and smooth muscle myosin heavy chain) were present in the peripheral, abluminal cells supporting their myoepithelial phenotype, while tumor cells lining true lumina were negative, consistent with their epithelial phenotype. However, tumor cells adjacent to spaces containing eosinophilic basement membrane-like material or basophilic stromal mucin showed myoepithelial differentiation, and stained similar to the peripheral, abluminal tumor cells. The expression of all markers appears to diminish in adenoid cystic carcinoma with solid areas and necrosis, as reported before. ${ }^{2,3}$ Solid variants of adenoid cystic carcinoma may be considered high grade, and are believed to be associated with worse prognosis. ${ }^{14,15}$ However, the role of these antibodies as prognostic markers needs further exploration.

The zonal pattern of immunostaining highlighting the 2-cell population in adenoid cystic carcinoma was not seen in polymorphous low-grade adenocarcinoma. Tumor cells in polymorphous low-grade adenocarcinoma were monomorphous containing round to oval, vesicular nuclei with open chromatin and small, distinct or indistinct nucleoli. These tumor cells did not express the markers suggestive of myoid/smooth muscle differentiation, consistent with previous reports. ${ }^{2,11,16,17}$ However, polymorphous low-grade adenocarcinoma expressed metallothionein, maspin, and p63, the latter two frequently and diffusely. Metallothionein, maspin, and p63 are known to be strongly expressed in simple epithelia, basal/stem cells and myoepithelial cells. Edwards et al ${ }^{3}$ reported p63 expression in all polymorphous low-grade adenocarcinoma, adenoid cystic carcinoma, and basal cell adenomas (100\%). They suggested an epithelial basal/stem/reserve cell origin for polymorphous low-grade adenocarcinoma, but since p63 also stains myoepithelial cells, myoepithelial histogenesis cannot be completely ruled out. Perez-Ordonez et $a l^{17}$ reported that all polymorphous low-grade adenocarcinoma (100\%) expressed $34 \beta$ E12 in their series. Similarly Gnepp et $a l$ and Regezi et $a l^{18,19}$ reported the expression of high molecular weight keratin in PLGA. High molecular weight keratin, $34 \beta \mathrm{E} 12$, and p63 are frequently used in prostate pathology to highlight the basal cells. Diffuse expression of p63 is seen in squamous, transitional, and basal cell carcinoma but not in adenocarcinoma. ${ }^{20}$ Thus, the expression of p63 in polymorphous low-grade adenocarcinoma may suggest basal/stem cell phenotype than glandular differentiation. Evidence for basaloid differentiation has also been demonstrated by electron microscopy in polymorphous low-grade adenocarcinoma. ${ }^{21}$ Indeed, expression of carcinoembryonic antigen, a hallmark of glandular differentiation, seen in most adenocarcinomas has rarely been demonstrated in polymorphous low-grade adenocarcinoma. ${ }^{17,18,22}$ The tumor cells in polymorphous lowgrade adenocarcinoma do not express proteins associated with myoid differentiation (smooth muscle actin, smooth muscle myosin heavy chain, calponin) or glandular differentiation (carcinoembryonic antigen) but express proteins associated with basal cells such as $34 \beta \mathrm{E} 12$, p63, maspin, and metallothionein. This appears to favor basaloid/ stem/reserve cell immunophenotypic features.

Hierarchical cluster analysis finds clusters of similar observations within a dataset. It is frequently used to analyze mRNA expression profiles of thousands of genes in DNA microarray studies. In recent years, this statistical tool has been applied to analyze immunohistochemical results on Formalinfixed paraffin-embedded tissues using multiple 
antibodies, and has demonstrated that tumors clustered into groups that correlated to their site of origin,${ }^{23}$ phenotype ${ }^{24,25}$ biologic potential, ${ }^{26}$ and prognosis. ${ }^{27-29}$ Our goal was to identify a cluster of markers that can be used in combination in a diagnostic immunohistochemical panel for differentiating adenoid cystic carcinoma from polymorphous low-grade adenocarcinoma. In this study, 47 tumors were immunostained with primary antibodies against six proteins, and their expressions were subclassified into three categories (negative, focal, and diffuse expression), generating a dataset comprising of $846(47 \times 6 \times 3)$ individual observations. Four markers, smooth muscle actin, calponin, smooth muscle myosin heavy chain, and metallothionein separated adenoid cystic carcinoma from polymorphous low-grade adenocarcinoma. Using these four markers, we analyzed the 47 tumors expecting them to fall into two distinct clusters, adenoid cystic carcinoma, and polymorphous lowgrade adenocarcinoma, and most samples did show expected results. Interestingly, four tumors did not cluster in their expected groups. ACC16 clustered with polymorphous low-grade adenocarcinoma. This tumor was reviewed by consultant oral pathologists who were blinded to the results of this study (not coauthors) and was reclassified as polymorphous low grade adenocarcinoma. PLGA4, PLGA5, and PLGA9 clustered with adenoid cystic carcinoma, and were reviewed by a consultant head and neck pathologist (not coauthor) who was also blinded to the results of this study. PLGA4 was reclassified as adenoid cystic carcinoma while PLGA5 and PLGA9 were reaffirmed to be polymorphous low-grade adenocarcinoma. PLGA5 had a predominantly fascicular architecture with a small tubulo-cystic component and a predominant spindle-cell component that showed myoid myoepithelial differentiation. PLGA9 had no distinguishing features when compared to other polymorphous low-grade adenocarcinoma. Thus, hierarchical cluster analysis suggested the correct diagnosis in 45 of 47 tumors $(95.7 \%)$.

It needs to be pointed out that none of the markers are specific for myoepithelial, basal or stem cells. Smooth muscle actin, smooth muscle myosin heavy chain, and calponin are also expressed by smooth muscle cells, proliferating fibroblasts, and blood vessels. Metallothionein is expressed in simple epithelia, basal cells, benign, and malignant mammary cells and tumors of various organs. Maspin is expressed by squamous and glandular epithelia, prostatic basal cells, endometrium, testicular germ cells and benign, and malignant mammary cells. p63 expression is seen in basal, squamous, and transitional cells. We also observed that only when more than one-third of the tumor cells were immunoreactive with smooth muscle actin, smooth muscle myosin heavy chain, calponin, and metallothionein, there was a significant association with adenoid cystic carcinoma. Staining reactions of lesser degrees were not distinctive of either tumor. Therefore, we recommend a high threshold for positive interpretation of immunohistochemical reaction with these markers in the differentiation of adenoid cystic carcinoma from polymorphous low-grade adenocarcinoma.

Most experts can differentiate adenoid cystic carcinoma from polymorphous low-grade adenocarcinoma using routine hematoxylin and eosin stains. However, diagnostic difficulties are encountered often. Immunohistochemical panels with multiple markers are a common practice in general surgical pathology and may lead to a false sense of confidence. Our results show that several markers do not improve diagnostic accuracy significantly when used in combination, as their immunoreactions are not complimentary, but virtually identical $(\kappa \leq 0.0035)$. Thus, using a panel of primary antibodies would not be any more helpful than using a single marker. In this study, smooth muscle actin provided the highest accuracy and positive predictive value for adenoid cystic carcinoma when compared to several other individual markers. Primary antibody to smooth muscle actin is available in most immunohistochemistry laboratories, and its use as a single agent may be more economical than the use of multiple markers. Smooth muscle actin may also be helpful in differentiating polymorphous low-grade adenocarcinoma from other salivary gland tumors of myoepithelial phenotype, for example, pleomorphic adenoma. However, we would like to caution that immunohistochemistry does not replace or override good morphologic examination, and should be used to support it.

In summary, although adenoid cystic carcinoma and polymorphous low-grade adenocarcinoma expressed several myoepithelial and basal/stem cells markers, the extent and pattern of expression were different in the two tumors. Diffuse expression of myoid myoepithelial markers (smooth muscle actin, calponin, smooth muscle myosin heavy chain) and metallothionein in tumor cells is highly predictive of adenoid cystic carcinoma. However, the use of multiple markers did not improve accuracy over using a single marker. A single marker, such as smooth muscle actin may be as informative as and more economical than a panel of multiple markers.

\section{Acknowledgements}

We thank Carl Allen, DDS, and John Kalmar, DMD, $\mathrm{PhD}$, Section of Oral and Maxillofacial Surgery \& Pathology, College of Dentistry, The Ohio State University, and Bayardo Perez-Ordonez, MD, Section of Head \& Neck Pathology, Toronto General Hospital, Toronto, Canada for their support and for their expert opinion on some of the cases. We thank Susie Jones for her help with immunohistochemistry. 


\section{References}

1 Castle JT, Thompson LD, Frommelt RA, et al. Polymorphous low grade adenocarcinoma: a clinicopathologic study of 164 cases. Cancer 1999;86:207-219.

2 Beltran D, Faquin WC, Gallagher G, et al. Selective immunohistochemical comparison of polymorphous low-grade adenocarcinoma and adenoid cystic carcinoma. J Oral Maxillofac Surg 2006;64:415-423.

3 Edwards PC, Bhuiya T, Kelsch RD. Assessment of p63 expression in the salivary gland neoplasms adenoid cystic carcinoma, polymorphous low-grade adenocarcinoma, and basal cell and canalicular adenomas. Oral Surg Oral Med Oral Pathol Oral Radiol Endod 2004;97:613-619.

4 Penner CR, Folpe AL, Budnick SD. C-kit expression distinguishes salivary gland adenoid cystic carcinoma from polymorphous low-grade adenocarcinoma. Mod Pathol 2002;15:687-691.

5 Cherian MG, Jayasurya A, Bay B-H. Metallothioneins in human tumors and potential roles in carcinogenesis. Mutat Res 2003;533:201-209.

6 Jin R, Bay BH, Chow VT, et al. Significance of metallothionein expression in breast myoepithelial cells. Cell Tissue Res 2001;303:221-226.

7 Cherian MG. The significance of the nuclear and cytoplasmic localization of metallothionein in human liver and tumor cells. Environ Health Perspect 1994;102(Suppl 3):131-135.

8 Nartey N, Cherian MG, Banerjee D. Immunohistochemical localization of metallothionein in human thyroid tumors. Am J Pathol 1987;129:177-182.

9 Zou Z, Anisowicz A, Hendrix MJ, et al. Maspin, a serpin with tumor-suppressing activity in human mammary epithelial cells. Science 1994;263:526-529.

10 Zarbo RJ, Prasad AR, Regezi JA, et al. Salivary gland basal cell and canalicular adenomas: immunohistochemical demonstration of myoepithelial cell participation and morphogenetic considerations. Arch Pathol Lab Med 2000;124:401-405.

11 Prasad AR, Savera AT, Gown AM, et al. The myoepithelial immunophenotype in 135 benign and malignant salivary gland tumors other than pleomorphic adenoma. Arch Pathol Lab Med 1999;123:801-806.

12 Weinberger PM, Yu Z, Haffty BG, et al. Molecular classification identifies a subset of human papillomavirus-associated oropharyngeal cancers with favorable prognosis. J Clin Oncol 2006;24:736-747.

13 Luna MA, Wenig BM. Polymorphous low-grade adenocarcinoma. In: Barnes L, Eveson JW, Reichart P, Sidransky D (eds). World Health Organization Classification of Tumors: Pathology and Genetics of Head and Neck Tumors. IARC Press: Lyon, France, 2005, pp 223-224.

14 Peel R. Diseases of the salivary glands. In: Barnes L (ed). Surgical Pathology of the Head and Neck, 2nd edn. M. Dekker: New York, NY, 2001, pp 699-701.

15 da Cruz Perez DE, de Abreu Alves F, Nobuko Nishimoto I, et al. Prognostic factors in head and neck adenoid cystic carcinoma. Oral Oncol 2006; 42:139-146.
16 Chen JC, Gnepp DR, Bedrossian CW. Adenoid cystic carcinoma of the salivary glands: an immunohistochemical analysis. Oral Surg Oral Med Oral Pathol 1988;65:316-326.

17 Perez-Ordonez B, Linkov I, Huvos AG. Polymorphous low-grade adenocarcinoma of minor salivary glands: a study of 17 cases with emphasis on cell differentiation. Histopathology 1998;32:521-529.

18 Gnepp DR, Chen JC, Warren C. Polymorphous lowgrade adenocarcinoma of minor salivary gland. An immunohistochemical and clinicopathologic study. Am J Surg Pathol 1988;12:461-468.

19 Regezi JA, Zarbo RJ, Stewart JC, et al. Polymorphous low-grade adenocarcinoma of minor salivary gland. A comparative histologic and immunohistochemical study. Oral Surg Oral Med Oral Pathol 1991;71: 469-475.

20 Di Como CJ, Urist MJ, Babayan I, et al. p63 expression profiles in human normal and tumor tissues. Clin Cancer Res 2002;8:494-501.

21 Norberg LE, Burford-Mason AP, Dardick I. Cellular differentiation and morphologic heterogeneity in polymorphous low-grade adenocarcinoma of minor salivary gland. J Oral Pathol Med 1991;20:373-379.

22 Anderson C, Krutchkoff D, Pedersen C, et al. Polymorphous low grade adenocarcinoma of minor salivary gland: a clinicopathologic and comparative immunohistochemical study. Mod Pathol 1990;3: 76-82.

23 Hsu FD, Nielsen TO, Alkushi A, et al. Tissue microarrays are an effective quality assurance tool for diagnostic immunohistochemistry. Mod Pathol 2002;15:1374-1380.

24 Au NHC, Cheang M, Huntsman DG, et al. Evaluation of immunohistochemical markers in non small cell lung cancer by unsupervised hierarchical clustering analysis: a tissue microarray study of 284 cases and 18 markers. J Pathol 2004;204:101-109.

25 Olsen SH, Thomas DG, Lucas DR. Cluster analysis of immunohistochemical profiles in synovial sarcoma, malignant peripheral nerve sheath tumor, and Ewing sarcoma. Mod Pathol 2006;19:659-668.

26 Zhang W, Hart J, McLeod HL, et al. Differential expression of the AP-1 transcription factor family members in human colorectal epithelial and neuroendocrine neoplasms. Am J Clin Pathol 2005; 124:11-19.

27 Bozcuk H, Gumus A, Ozbilim G, et al. Cluster analysis of p-glycoprotein, c-erb-B2 and P53 in relation to tumor histology strongly indicates prognosis in patients with operable non small cell lung cancer. Med Sci Monit 2005;11:HY11-HY20.

28 Lyall MS, Dundas SR, Curran S, et al. Profiling markers of prognosis in colorectal cancer. Clin Cancer Res 2006;12:1184-1191.

29 Makretsov NA, Huntsman DG, Nielsen TO, et al. Hierarchical clustering analysis of tissue microarray immunostaining data identifies prognostically significant groups of breast carcinoma. Clin Cancer Res 2004;10:6143-6151. 